\title{
EFFECTS OF COMPLEX MEDICAL REHABILITATION TREATMENT ON LIGAMENT INSTABILITY IN PATIENTS WITH KNEE OSTEOARTHRITIS
}

\section{Chiriac Marius Sorin ${ }^{12}$, Chirila Sergiu², Hangan Tony'2, Zamfirescu Mihaela².}

${ }^{1}$ Balneal Sanatorium and Rehabilitation Techirghiol

${ }^{2}$ Faculty of Medicine, University "Ovidius" of Constanta

Marius Sorin Chiriac

Balneal Sanatorium and Rehabilitation Techirghiol, Victor Climescu nr.34-40, Techirghiol, Romania email: chiriacsorin2002@yahoo.co.uk phone: +4022469436

\begin{abstract}
Knee osteoarthritis represents one of the most frequent conditions in the elderly population. This condition affects the whole joint, having an important impact on the patient's quality of life.

In this study, we evaluate the effect that medical rehabilitation treatment has on ligament instability in patients with knee osteoarthritis.

Material and method: we evaluate, using specific tests, the anterior, posterior, lateral and medial stability in patients that undergo rehabilitation treatment. Evaluations were done initially, at 6 months after the first admission and at 12 months after the first admission. We compare the results in order to evaluate the impact the complex therapies that are applied have.

Results: in all cases, except posterior instability, there is a good effect in lowering the percentage of patients with positive tests after the first round of rehabilitation treatment. At six month after the second round of rehabilitation treatment (12-month evaluation) the results show a slight, non-statistically significant improvement over the 6-month evaluation.
\end{abstract}

Keywords: knee osteoarthritis, evolution, ligament instability, rehabilitation treatment

\section{Introduction}

Osteoarthritis represents a degenerative condition, which involves the articulations. This is characterized by the destruction of the cartilage and modifications that affect all anatomical elements of the joint (1). The knee osteoarthritis is the most frequently met site (2) and is considered a disease of the whole joint, not only of the cartilage but also meniscus, ligaments, and muscles (3). This leads to severe pain, joint instability and a decreased quality of life (4)

The main factor that leads to knee osteoarthritis is aging (5), probably as a consequence of degenerative processes that take place.

Knee instability is found in a high percentage of the patients with knee osteoarthritis (6), most of the times being self-reported. Ligaments play an important role in the stability of the knee joint. The ligaments control the range of motion and also provide mechanical reinforcement (7). There are four major ligaments that provide this, anterior cruciate ligament, posterior cruciate ligament, lateral collateral ligament and medial collateral ligament (8). The anterior cruciate ligament and posterior cruciate ligament prevent the antero-posterior sliding of the bones, while the lateral collateral ligament and medial collateral ligament prevent the side-to-side movement. 
The primary objective of non-surgical interventions in patients with knee osteoarthritis is to improve the quality of life by improving the joint stability, functionality, reduce pain and prevent progression of osteoarthritis (9).

In general, studies that evaluate nonsurgical treatment of osteoarthritis focus on pain relief (10) and function.

In this study, we assess the effect that complex medical rehabilitation treatment has on knee instability.

\section{Material and method}

The study was conducted on 102 patients diagnosed with knee osteoarthritis in Balneal Sanatorium and Rehabilitation Techirghiol from 01.07 .2017 to 01.08 .2018 out of which 58 completed the study. The rest of the patients withdrawn or lost in follow-up. The patients were evaluated before the first intervention, at the 6 months readmission, and at 12 months readmission. There were three evaluations for each patient that completed the study.

The average age of the patients was 63.88 years, with a standard deviation of 10.42 years. Most of the patients were between 60 and 69 years old, and $83 \%$ were women, mostly coming from urban areas $(62 \%)$.

We evaluated the influence of complex rehabilitation and physical therapy interventions on knee stability. The tests that we used were:

- Anterior stability: Lachman Test and Anterior Drawer Test

- Posterior stability: Reversed Lachman Test and Posterior Drawer Test

- Medial stability: Swain Test and Valgus Stress Test

- Lateral stability: Varus Stress Test

The rehabilitation treatment of every patient was: mud baths from Techirghiol lake, hydrotherapy with saltwater, kinesiotherapy for the knee, electrotherapy, sonotherapy, laser therapy, magnetotherapy. This program was made by the patients every day for 10 days of treatment.

We compared the proportion of patients with positive or negative results for each time of measurement. To identify if there are statistically significant modifications of the proportion of patients with positive results, we ran Cochrane's Q test with post-hoc analysis using Dunn's procedure with a Bonferroni correction for multiple comparisons. For Cochrane's Q test, we report the exact level of significance. For pairwise comparisons, adjusted p-values are presented. We used a cut-off point for the significance of $p=0.05$. For analysis, we used IBM SPSS Statistics 25.

\section{Results}

\section{Anterior stability - Lachman Test}

Lachman Test was positive in $74.1 \%$ of the patients at the initial evaluation. At the six month evaluation, the percentage of patients with a positive result was $48.3 \%$, and at the 12 -month evaluation, their proportion was $36 \%$ (Figure 1). The results are statistically significant $\left(\chi^{2}(2)=31.58, p<0.001\right)$.

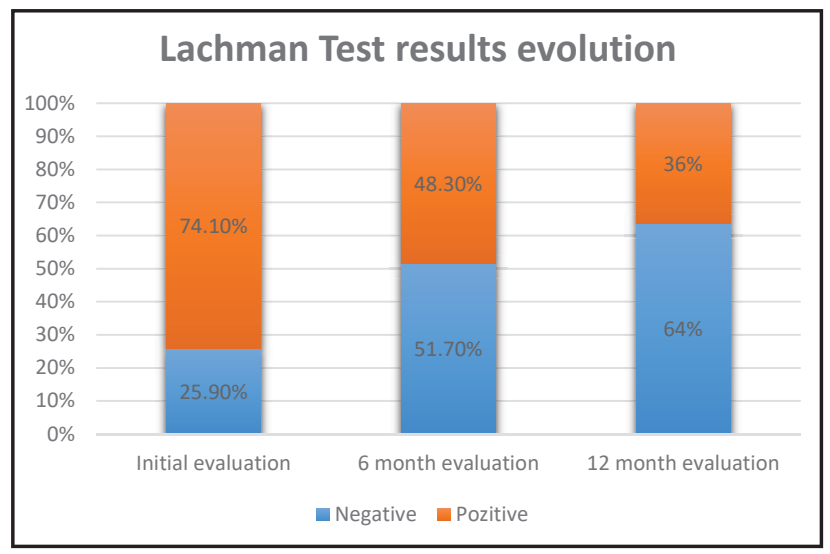

Figure 1 Lachman test results in evolution

Compared to the initial percentage of patients with a positive Lachman test, there was a statistically significant decrease in the percentage positive after six months $(\mathrm{p}<0.001)$ and after 12 months $(p=0.001)$. There was no statistically significant difference in the percentage of patients with a positive Lachman test between 6-month and 12-month evaluation ( $\mathrm{p}=0.24)$ (Figure 2$)$. 


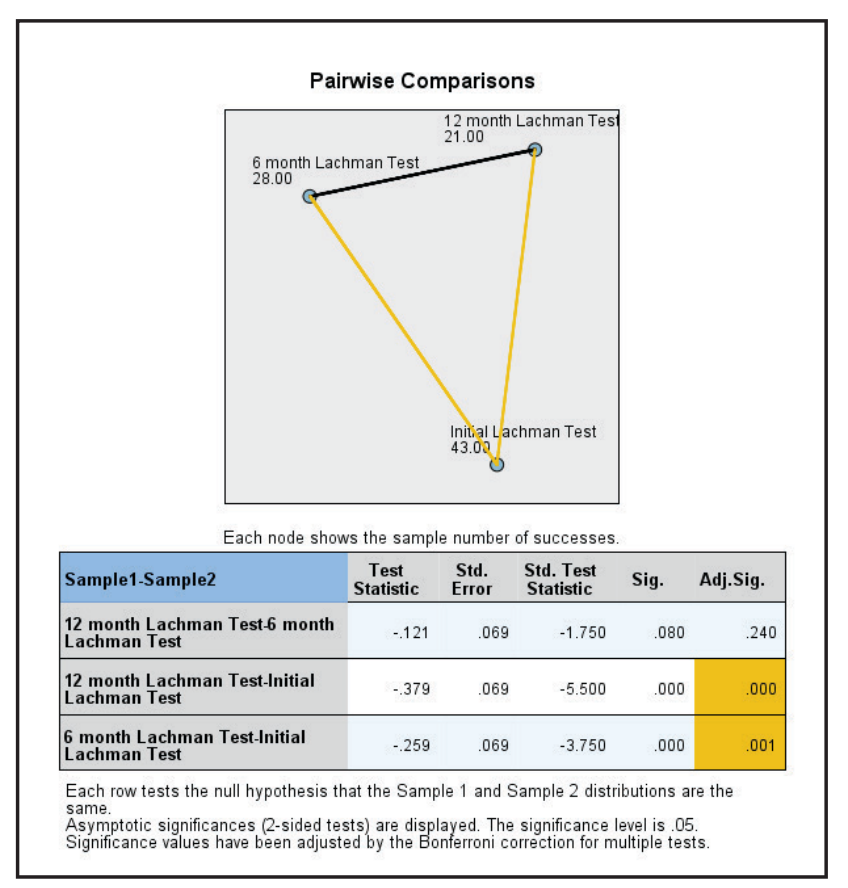

Figure 2 Pairwise comparison Lachman test

\section{Test \\ Anterior Stability - Anterior Drawer}

Anterior Drawer Test was positive, at the initial evaluation, for $65.5 \%$ of the patients. At the intermediary evaluation, done after six months from the first admission, the percentage of patients with a positive result was $41.40 \%$ and dropped, at the 12-month evaluation to $31 \%$ (Figure 3). The differences were statistically significant $\left(\chi^{2}(2)=28.73, \mathrm{p}<0.001\right)$

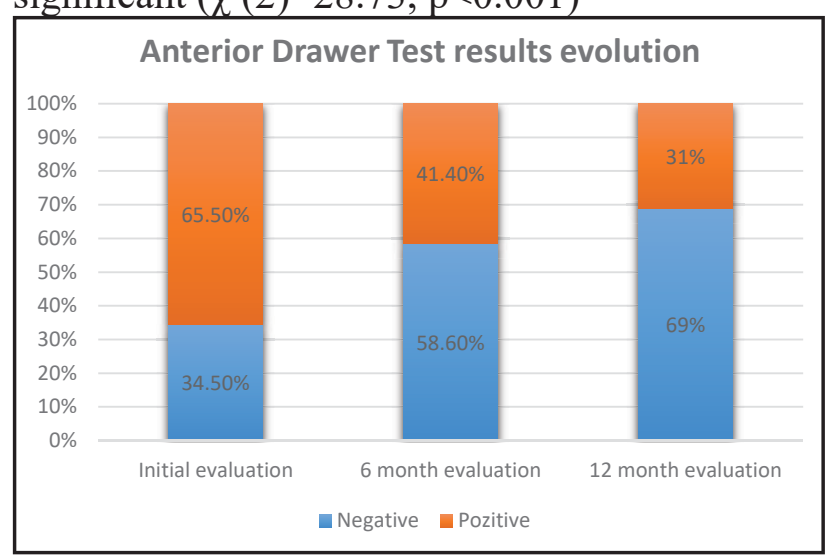

Figure 3 Anterior Drawer Test results in evolution

The pairwise comparison revealed the fact that there were statistically significant differences between results obtained at initial evaluation and 6-month evaluation $(\mathrm{p}<0.001)$ and between initial evaluation and 12-month evaluation $(p<0.001)$. No statistically significant difference was observed between 6-month evaluation and 12-month evaluation $(\mathrm{p}=0.352)$.

Posterior stability - Posterior Drawer Test

For testing the posterior stability, we used two tests. The Posterior Drawer Test and Reversed Lachman Test.

The results for the Posterior Drawer Test at initial evaluation identified $50 \%$ of the patients with a positive result. The percentage of patients with positive results at 6 -month evaluation was $48.3 \%$ and at 12 -month evaluation, $48.3 \%$ of the patients had positive results (Figure 4). There were no statistically significant differences $\left(\chi^{2}(2)=2, p=1\right)$.

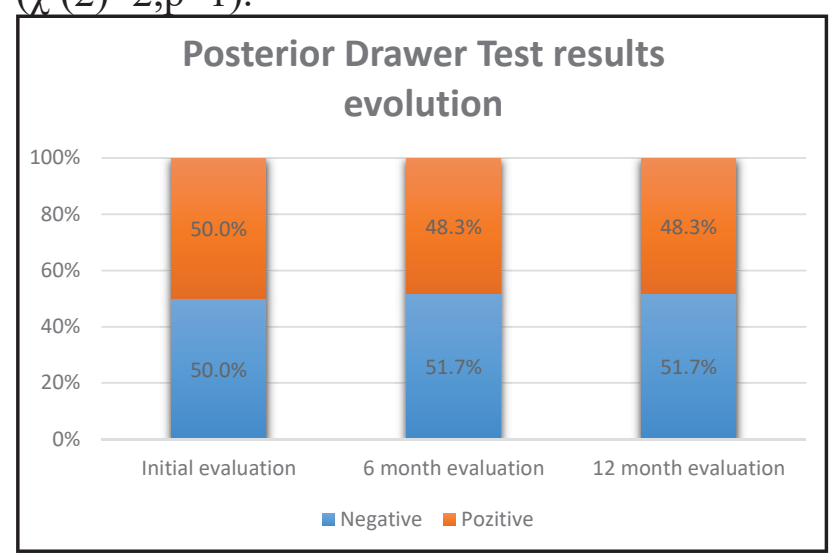

Figure 4 Posterior Drawer Test results in evolution

\section{Test \\ Posterior stability - Reversed Lachman}

The Reversed Lachman Test is used for assessing the posterior cruciate ligament. The result was positive for $19 \%$ of the patients at the initial evaluation. At six month evaluation, $13.8 \%$ of the patients had a positive result, and at 12 -month evaluation, $15.5 \%$ of the patients were recorded with a positive test (Figure 5). The differences are not statistically significant $\left(\chi^{2}(2)=1.75, \mathrm{p}=0.556\right)$.

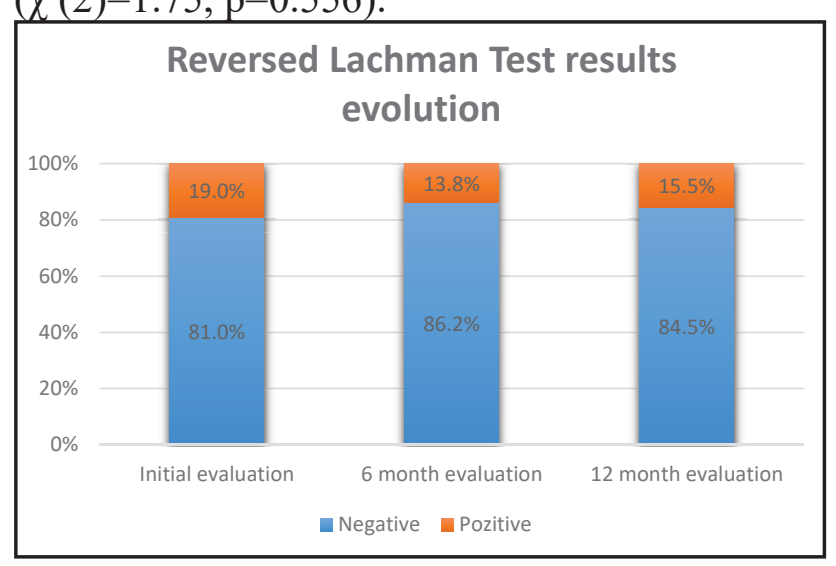

Figure 5 Reversed Lachman Test results in evolution 


\section{Medial stability test - Swain Test}

In order to evaluate the medial stability, we performed Swain Test and Valgus Stress Test.

At the initial evaluation, using the Swain Test, $31 \%$ of the patients presented with a positive test. This percentage dropped significantly at the six-month evaluation to $13.8 \%$. At the 12 month evaluation, the percentage of patients with positive Swain Test continued to drop, reaching $10.3 \%$ (Figure 6). The result is statistically significant $\left(\chi^{2}(2)=20.67, \mathrm{p}<0.001\right)$.

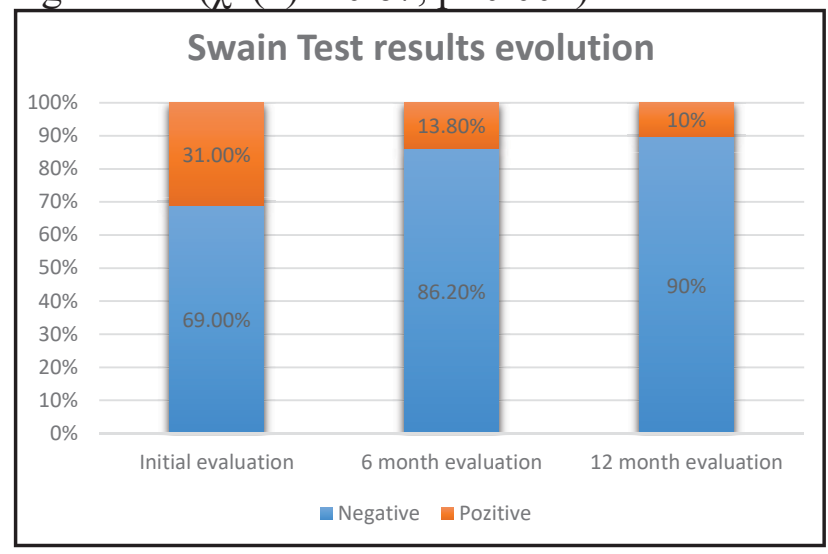

Figure 6 Swain Test results in evolution

Pairwise comparison identified the fact that between the initial evaluation and the evaluations conducted at six and 12 months, the difference is statistically significant $(p<0.001)$. Still, there is no statistically significant difference between the six-month evaluation and the 12-month evaluation $(\mathrm{p}=1)$, as the proportion of patients with positive results is almost similar.

\section{Medial stability test - Valgus Stress Test}

For Valgus Stress Test we observed the same tendency, with an initial positive test for $67.2 \%$ of the patients. The percentage of positive tests dropped to $41.4 \%$ at the six-month evaluation and dropped further to $36.2 \%$ at the 12-month evaluation (Figure 7). The differences between the three proportions are statistically significant $\left(\chi^{2}(2)=24.26, \mathrm{p}<0.001\right)$.

Pairwise comparison identified the fact that between the initial evaluation and the evaluations conducted at six and 12 months using the Valgus Stress Test, the difference is statistically significant $(p<0.001)$. There is no statistically significant difference between the six-month evaluation and the 12-month evaluation $(p=1)$.

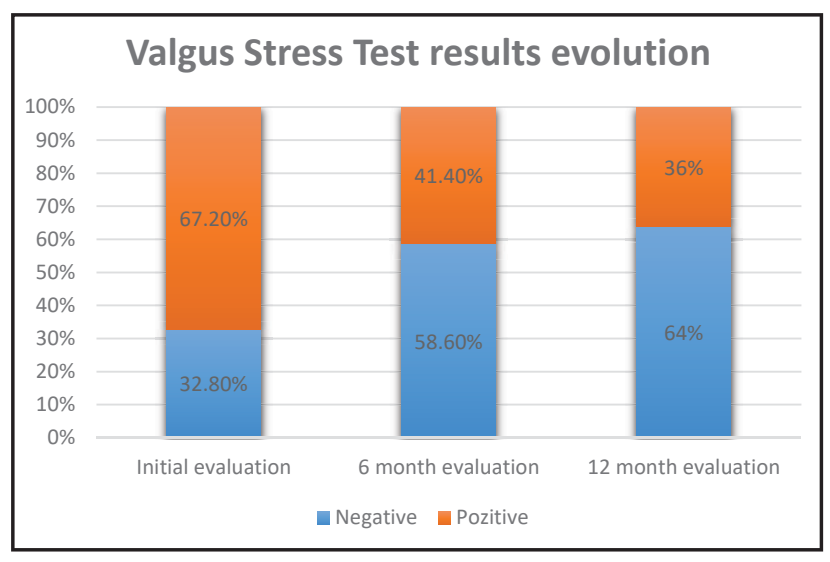

Figure 7 Valgus Stress Test results evolution

\section{Lateral Stability - Varus Stress Test}

Evaluation of the lateral stability with the Varus Stress Test found that, at the initial moment, $70.7 \%$ of the patients had a positive outcome. This percentage dropped considerably at the 6-month evaluation, to $46.6 \%$, and dropped even more at 12-month evaluation, to less than 40\% (39.7\%) (Figure 8). Cochrane's Q test result indicates a statistically significant difference $\left(\chi^{2}(2)=26.8, p<0.001\right)$.

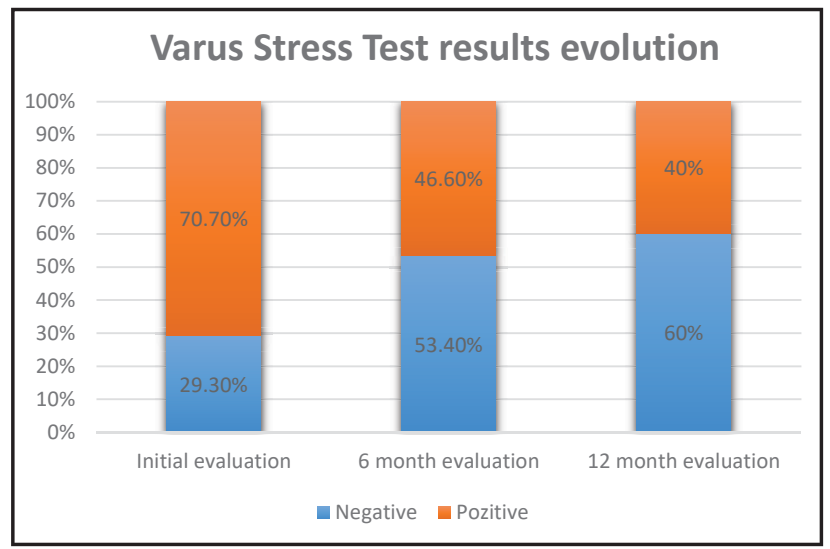

Figure 8 Varus Stress Test results in evolution

The pairwise analysis identified statistically significant differences of the proportion of patients with positive results between initial evaluation and 6-month evaluation $(p<0.001)$ and initial evaluation and 12-month evaluation $(p<0.001)$. Between the evaluations conducted at 6 months and 12-month evaluation, the difference in proportion was not statistically significant $(\mathrm{p}=0.82)$. 


\section{Discussions}

According to the results described, in the case of anterior stability evaluation, both tests used to evaluate the status of the knee, Lachman Test and Anterior Drawer Test showed improvements, with decreasing percentages of positive results following the first admission into the rehabilitation institution. After the second admission, which took place at a six months interval after the first one, the improvements were less important, the pairwise analysis showing differences that were not statistically significant.

For posterior stability, we used the Reversed Lachman Test and Posterior Drawer Test. Both of the tests showed minor improvements, the result is in both cases not statistically significant. Thus, the intervention did not influence the posterior stability of the osteoarthritic knee.

For the medial stability, evaluated through the Valgus Stress Test there was a statistically significant improvement, with a decrease of the percentage of patients with positive test result following the first rehabilitation intervention, but with no further improvement after the second intervention. In the case of Swain Test, the results are similar, showing and improvement over time, especially after the first course of rehabilitation.

Lateral stability, evaluated through Varus Stress Test also shows improvements after the first course of rehabilitation intervention, which is statistically significant. The effect improves further (but without being statistically significant) after the second course of treatment.

\section{Conclusions}

Complex rehabilitation programs are effective in improving knee stability in patients with osteoarthritis.

Posterior stability is not influenced by the complex rehabilitation program followed by the patients in this study.

\section{References}

1. Hutton CW. Osteoarthritis: the cause not result of joint failure? Ann Rheum Dis. 1989;48(11):958-61.

2. Lawrence RC, Felson DT, Helmick CG, Arnold LM, Choi H, Deyo RA, et al. Estimates of the prevalence of arthritis and other rheumatic conditions in the United States: Part II. 2008;58(1):26-35.

3. Heidari B. Knee osteoarthritis prevalence, risk factors, pathogenesis and features: Part I. Caspian J Intern Med. 2011;2(2):205-12.

4. Abbott JH, Usiskin IM, Wilson R, Hansen $\mathrm{P}$, Losina E. The quality-of-life burden of knee osteoarthritis in New Zealand adults: A model-based evaluation. PloS one. 2017;12(10):e0185676.

5. Zhang Y, Jordan JM. Epidemiology of osteoarthritis. Clin Geriatr Med. 2010;26(3):355-69.

6. van der Esch M, van der Leeden M, Roorda LD, Lems WF, Dekker J. Predictors of selfreported knee instability among patients with knee osteoarthritis: results of the Amsterdam osteoarthritis cohort. Clinical rheumatology. 2016;35(12):3007-13.

7. Blalock D, Miller A, Tilley M, Wang J. Joint instability and osteoarthritis. Clin Med Insights Arthritis Musculoskelet Disord. 2015;8:15-23.

8. Standring S, Borley NR. Gray's Anatomy: The Anatomical Basis of Clinical Practice: Churchill Livingstone/Elsevier; 2008.

9. Vaishya R, Pariyo GB, Agarwal AK, Vijay V. Non-operative management of osteoarthritis of the knee joint. J Clin Orthop Trauma. 2016;7(3):170-6.

10. Bjordal JM, Johnson MI, Lopes-Martins RA, Bogen B, Chow R, Ljunggren AE. Shortterm efficacy of physical interventions in osteoarthritic knee pain. A systematic review and meta-analysis of randomised placebocontrolled trials. BMC musculoskeletal disorders. 2007;8:51. 